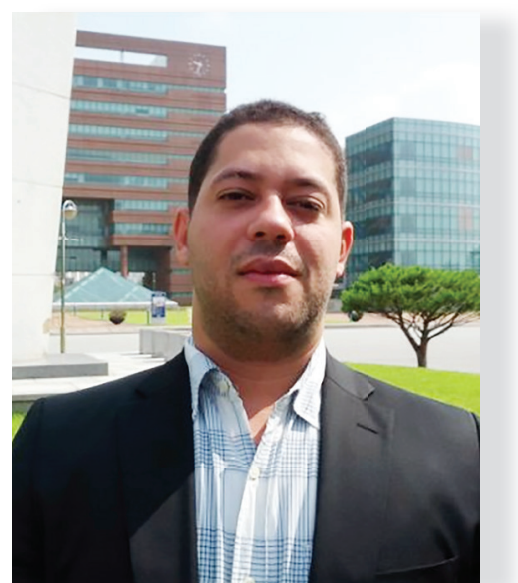

Dr. Víctor González Holguín

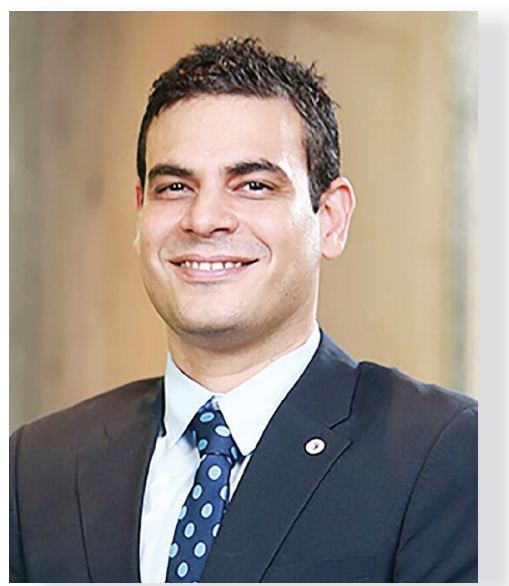

Ing. Julio Ferreira Tavera"

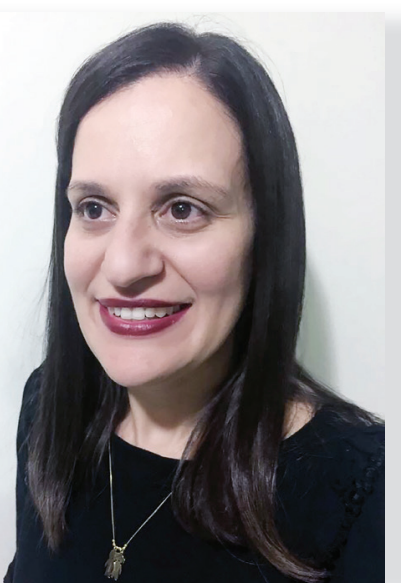

Ing. Ana Barranco López"'

\title{
Desarrollo de habilidades blandas y el uso del Sistema de Gestión del Aprendizaje en la elaboración de proyectos prácticos en una asignatura introductoria de Ingeniería Telemática
}

\section{Development of soft skills and the use of the Learning Management System in the preparation of practical projects in a telematics engineering introductory course}

Recibido: 07-04-17

Aprobado: 26-09-17

\section{Resumen}

La práctica de la ingeniería tiene un alto uso de proyectos. Los proyectos requieren el trabajo de equipos multidisciplinarios y la comunicación constante para poder ser exitosos. En la enseñanza de ingeniería se deben utilizar métodos que permitan acercar a los estudiantes con la forma de trabajo del campo laboral en el que se desempeñarán, por lo tanto, para este estudio se ha implementado la metodología de Aprendizaje Orientado a Proyectos con el fin de establecer una conexión entre las competencias técnicas y las habilidades blandas de los estudiantes. Se utilizó una asignatura introductoria en el programa de estudio de Ingeniería Telemática y se desarrollaron proyectos relacionados al Internet de las Cosas para conectar las capacidades técnicas con habilidades blandas como comunicación oral, comunicación escrita y trabajo en equipo. El uso de esta metodología en conjunto con un Sistema de Gestión del Aprendizaje permitió una mejora significativa en la escritura de reportes técnicos y en la habilidad para presentar y defender su proyecto. Es decir, se pudo alinear el desarrollo técnico con las habilidades blandas previamente mencionadas. Al mismo tiempo, se obtuvo un alto índice de satisfacción por parte de los estudiantes en el desempeño de los proyectos superando las expectativas iniciales de los mismos estudiantes.

*Dr. Víctor Manuel Gonzalez Holguín: Doctor en Tecnología de la Información y Telecomunicación por Korea Advanced Institute of Science and Technology (KAIST). Es profesor investigador a tiempo completo en la Pontificia Universidad Católica Madre y Maestra y director del departamento de Telemática. Además, se ha desempeñado como revisor invitado en distintas revistas y conferencias académicas internacionales. Para contactar al autor: victorgonzalez@pucmm.edu.do

**Ing. Julio Augusto Ferreira Tavera: Ingeniero en Electrónica, con especialidad en instrumentación y automatización de procesos. Master en Energías Renovables. Actualmente Decano de la Facultad de Ciencias e Ingeniería de la Pontificia Universidad Católica Madre y Maestra. Miembro de las sociedades de Energía, Sistemas de Control, Automatización y Robótica del Instituto de Ingenieros Eléctricos y Electrónicos (IEEE). Para contactar al autor: JulioFerreira@pucmm.edu.do

***Ing. Ana María Barranco López: Ingeniera Civil con maestría en Ingeniería Ambiental y Especialista en Pedagogía Universitaria. Experiencia profesional en el área de especialización en la Ingeniería Civil y con más de una década de labor docente a nivel universitario en el Depto. de Ingeniería Civil en la Pontificia Universidad Católica Madre y Maestra. Para contactar a la autora: abarranco@pucmm.edu.do 


\begin{abstract}
Projects are widely used in the practice of engineering. Most projects required multidisciplinary teams and constant communication between actors for their success. The education system should use pedagogical methods that are align to the practice of the field, in our case, of engineering. This study implemented the Project Base Learning to establish a link between the hard skills and soft skilled developed in an introductory course in the bachelor program of Information and Telecommunication Engineering. The projects focused on the concept of Internet of Things to develop on students the soft skills of communication (written and oral) and teamwork. The methodology used in the course along with a Learning Management System allowed a significant improvement in the technical reports written by the students and on their presentation skills of the projects developed in the course. We were able to align de hard skills developed with the needed soft skills mention above. This implementation resulted in a high-level satisfaction from the students on the projects they developed, overpassing their initial expectations.
\end{abstract}

\title{
Palabras clave
}

Aprendizaje Orientado a Proyectos; habilidades blandas; internet de las cosas; sistema de gestión de los aprendizajes

\section{Keywords}

Project based learning; soft skills; internet of things; learning management system

\section{Introducción}

Los programas de Ingeniería en la Pontificia Universidad Católica Madre y Maestra (PUCMM) requieren que los estudiantes realicen un Trabajo de Fin de Grado (TFG) para poder completar sus estudios. En el último año de la carrera los estudiantes deben realizar un proyecto relevante al área de estudio, el cual puede ser de investigación o de integración tecnológica. Un jurado formado por profesores y profesionales del área evalúan cada proyecto en los siguientes componentes: desarrollo técnico, reporte escrito y presentación oral.

En las presentaciones los jurados han detectado una carencia en las habilidades de comunicación oral y escrita de los estudiantes de último año de la carrera de Ingeniería Telemática en el Campus Santo Tomás de Aquino. Esta debilidad evidenciada contradice con el perfil de egreso de la Universidad.

En la práctica de la ingeniería son tan importantes las competencias técnicas de la carrera como las llamadas habilidades blandas. Estas son habilidades no técnicas que se han determinado como necesarias en el ambiente laboral, son transversales en las competencias del individuo y están más relacionadas a la personalidad, actitud y comportamiento de cada persona (Murti, 2014). De acuerdo con Ortega, Febles y Estrada: "la estrategia que se aplique debe contemplar el desarrollo tecnológico, acompañado de las nuevas estrategias de aprendizaje que el mundo moderno impone." (2016, p41)

De las distintas habilidades blandas, los empleadores y las agencias de acreditación requieren que en los programas de estudio de ingeniería se desarrolle el trabajo en equipo, así como la comunicación oral y escrita (Mills \& Treagust 2003). Estas competencias son tan importantes que tienen un efecto en la percepción que puede tenerse de un ingeniero con respecto a sus habilidades técnicas. Aquellos que tienen mayores habilidades de comunicación son percibidos con mayor capacidad técnica que los que tienen pocas, teniendo eso incidencia en la facilidad de encontrar empleo (Dahm, Farrell, \& Ramachandran, 2015).

Podemos establecer que las habilidades de trabajo en equipo y comunicación oral y escrita son primordiales para la práctica de un ingeniero. Estas se deben desarrollar de forma transversal en el programa de estudio desde la primera asignatura de la carrera (Pavlakis \& Kelley, 2016; Yalvac, Smith, Troy, \& Hirsch, 2007). Muchos estudiantes de ingeniería se resisten a trabajar en equipo o a escribir y piensan que estas habilidades no son necesarias para el ingeniero, siendo esto una concepción errada (Dahm, et al, 2015). Palma, Miñán y Ríos (2011) citando a Maffioli y Giuliano (2003) consideran que:

en la sociedad del conocimiento del nuevo milenio el perfil de un buen ingeniero debe basarse en la capacidad y voluntad de aprender, el conocimiento sólido de las ciencias naturales básicas y el buen conocimiento de algún campo de la tecnología, además de los valores humanos generales. Por otra parte, tiene que estar preparado para el aprendizaje permanente y también debe poseer una buena comunicación y trabajo en equipo. Las competencias técnicas no son suficientes en el mundo actual. (p. 2553) 
La demanda en el mercado laboral global de habilidades sociales como las mencionadas tuvo un crecimiento de un $24 \%$ desde 1980 hasta el 2012 y se estima que esta demanda solo seguirá creciendo (Deming, 2015). Debido a los cambios en tecnologías, las habilidades técnicas deben ser integradas con la habilidad de poder comunicarse con diferentes actores o público y trabajar en equipos multidisciplinarios. Los profesionales que tengan una alta integración de habilidades sociales como las mencionadas y competencias técnicas, tienen menor riesgo de ser sustituidos por procesos automáticos dadas las limitaciones de interacción que existe con las computadoras en la actualidad (David, 2015; Deming, 2015; Frey \& Osborne, 2017).

En la búsqueda de trabajo, las habilidades sociales de comunicación y trabajo en equipo determinan el candidato a contratar (Dahm et al., 2015). Estas habilidades son tan importantes que sin importar el alto nivel técnico que pueda tener un profesional, el mal manejo en comunicación o trabajo en equipo pueden perjudicar su desarrollo profesional. Inclusive, la habilidad de comunicación oral y escrita tiene una alta correlación al éxito profesional de una persona en el mundo corporativo (Robles, 2012).

El objetivo principal de este trabajo es desarrollar en los estudiantes las competencias de comunicación oral y escrita y otras competencias blandas como trabajo en equipo a través de la implementación de la metodología de Aprendizaje Orientado a Proyectos en una asignatura introductoria a la carrera de Ingeniería Telemática.

En las siguientes páginas se expone los conceptos fundamentales del Aprendizaje Orientado a Proyectos; luego se describe y se justifica la metodología utilizada, tanto su implementación como su evaluación. Porúltimo, se presentan los resultados de la implementación, las implicaciones encontradas y las conclusiones más relevantes obtenidas.

\section{Aprendizaje Orientado a Proyectos (AOP)}

En la práctica de la ingeniería un proyecto se utiliza como una unidad de trabajo. Este puede variar en complejidad, tiempo, escala y/o presupuesto. Los proyectos pueden tener diferencias significativas en las dimensiones expuestas, pero estarán relacionados en el uso de los fundamentos teóricos y las técnicas de la especialización en una disciplina de ingeniería (Mills \& Treagust 2003).

La utilización de proyectos para el aprendizaje se ha documentado en la educación superior de ingeniería por más de cien años (Yam \& Rossini, 2010). El Aprendizaje Orientado a Proyectos (AOP) forma parte de la teoría constructivista del aprendizaje. El AOP se puede ver como la suma de la subdivisión de varios problemas. Al mismo tiempo, el uso de esta metodología acerca el trabajo de los estudiantes en la universidad a la práctica profesional.

Una asignatura que esté dirigida al AOP permite la incorporación de otros métodos de enseñanzaaprendizaje para mejorar el proceso en los estudiantes asegurando la inclusión de distintas formas de aprendizaje que puedan tener los estudiantes (Felder, 1993). Los estudiantes que participan dentro de esta metodología generalmente tienen mayor motivación en los temas disciplinares y desarrollan sus habilidades sociales (Mills \& Treagust 2003). Esto se debe a que el desarrollo exitoso de un proyecto necesita que los estudiantes demuestren habilidades de comunicación, trabajo en equipo, gestión del tiempo y liderazgo (Davcev, Stojkoska, Kalajdziski, \& Trivodaliev, 2016). El desarrollo de estas habilidades son fundamentales para cualquier profesional (Dahm et al., 2015; Mills \& Treagust 2003). Las agencias de acreditación académica exigen el desarrollo de estas habilidades en los programas académicos que acreditan (DePiero \& Slivovsky, 2007; Sharma, Steward, Ong, \& Miguez, 2016) y el mercado laboral global también exige estas competencias. (Deming, 2015).

EI AOP ayuda al desarrollo de las habilidades blandas y las relaciona con las habilidades técnicas que un estudiante debe desarrollar dentro de su área disciplinar. Esta metodología se puede implementar de distintas formas. Las implementaciones con mayor impacto tienden a incorporar una competencia interna de los proyectos finales al curso (Krithivasan, Shandilya, Arya, Lala, Manavar, Patii \& Jain, 2014). Esta estrategia trata los diferentes temas teóricos y prácticos y el estudiante implementa lo aprendido en ambos casos para la creación de un proyecto (Davcev et al., 2016). En este tipo de implementación se debe considerar la dificultad del proyecto a proponer y el tiempo que se otorga para la entrega del mismo (Calnon, Gifford \& Agah, 2012; Jadhav, Mulla \& Jadhav, 2017). El agregar el factor de competencia al proyecto promueve la motivación intrínseca del estudiante a la asignatura, incrementando la creatividad del estudiante hacia el proyecto (Hernando, Galán, Navarro \& RodriguezLosada, 2011; Neebel, Merkel \& Wong, 2013).

El desarrollo de un proyecto en la metodología de AOP promueve que el estudiante deba buscar información para la elaboración del mismo. Esto implica que el estudiante debe leer y sintetizar textos relacionados al tema elegido. Al finalizar, debe realizar una síntesis escrita que fundamente de forma apropiada el proyecto. 
El desarrollo de la comunicación escrita es esencial para poder realizar dicha fundamentación descriptiva del tema. La habilidad de escritura es un acto social que pone como prioridad al público a quien está dirigido, adaptándose a las normas establecidas por el sistema social en el cual se desenvuelve el escritor y permitiendo demostrar lo aprendido. "En el ámbito universitario, aprender a escribir se ha convertido en un proceso de apropiación y manejo experto de las prácticas discursivas en las que se difunde y legitima el modo de pensamiento de un ámbito disciplinar determinado". (Alvarez \& Boillos, 2015, p. 55).

Con el uso de un Sistema de Gestión del Aprendizaje (SGA) se puede ofrecer contenido especializado para ayudar a los estudiantes en los diferentes temas que deben abordar para su proyecto, utilizando una forma de representar el contenido adaptado al estilo de aprendizaje del estudiante (Nafea, Maglaras, Siewe, \& Shehab, 2015). Esta herramienta también facilita la implementación de un Aprendizaje Orientado a Proyectos y favorece la comunicación del profesor con los estudiantes y entre los estudiantes (Krithivasan et al., 2014; Lou \& Kim MacGregor, 2004).

\section{Metodología}

La metodología a utilizar está basada en el Aprendizaje Orientado a Proyectos con un modo de integrar exposiciones de temas por expertos en el área, sesiones prácticas para trabajo en equipo y la utilización de un SGA como soporte para la revisión de literatura con contenidos apropiados a los temas y a su vez, este sistema de gestión es utilizado para la resolución de problemas.

La utilización de estas distintas metodologías nos permite alinear las diferentes dimensiones de estilo de aprendizaje que pueden tener los estudiantes, ayudando a mantener el interés en la asignatura y en la carrera (Felder, 1993). Además, promueve las ciencias y tecnologías mostrando la relevancia que estas tienen en sus vidas y sus intereses personales (Porter \& Simon, 2013).

Se reestructuró el programa de la primera asignatura de la carrera de Ingeniería Telemática para poder utilizar una combinación de metodologías que incentivan el interés de los estudiantes en el área de estudio y al mismo tiempo, desarrollan las habilidades de trabajo en equipo y comunicación oral y escrita.

Se utilizó la asignatura de Introducción a Sistemas de Computación, la cual está programada para el tercer periodo académico de los estudiantes cursando el programa de estudio de Ingeniería Telemática en la
Pontificia Universidad Católica Madre y Maestra. El promedio de estudiantes que cursan la asignatura en este periodo es de 20 estudiantes. Para el periodo 2016-2017-3 se inscribieron 21 estudiantes. El tamaño de la muestra es representativo a la población de la carrera, la cual tenía en el momento del estudio 175 estudiantes inscritos y entran aproximadamente 18 estudiantes nuevos por año en promedio.

El curso está diseñado para impartirse en un periodo de 10 semanas de clases y una semana adicional para los exámenes finales. El programa se dividió en 6 módulos, y en cada uno se invitó a un experto en el área para que expusiera su experiencia profesional y académica. Dos módulos se desarrollaron en cuatro sesiones de clase y cuatro módulos en dos sesiones de clase, cada sesión de clase era de 100 minutos.

Se utilizó la plataforma Moodle 3.0 como SGA, ya que es la herramienta de gestión de aprendizaje proporcionada por la Universidad. En la plataforma se colocaron todos los recursos didácticos utilizados por los expertos. También fueron administradas a través del sistema las pruebas cortas para medir el desempeño en cada módulo. De la misma manera, la plataforma también fue utilizada para impartir el examen final. La asignación y entrega de los trabajos escritos se realizaron por la misma plataforma. Desde el inicio de la asignatura los estudiantes tuvieron acceso a las rúbricas que se utilizarían para evaluar las diferentes competencias al finalizar el periodo académico desde la plataforma. La utilización de este recurso posibilitó que los estudiantes pudieran recibir una retroalimentación de su trabajo en el momento que el profesor realizó la evaluación siguiendo los criterios establecidos en la asignación de cada trabajo.

Para desarrollar la habilidad de comunicación escrita seguimos una modificación al método desarrollado por Yalvac (Yalvac et al., 2007). Se empezó el curso con un taller de 90 minutos con el tema de "Formas de escribir en Ciencias e Ingeniería". Luego del taller, se entregaron las rúbricas a utilizar para evaluar el trabajo final y se socializaron con los estudiantes para aclarar cualquier duda. Se asignaron cuatro trabajos escritos de los cuales dos fueron individuales y dos fueron grupales. Cada trabajo tuvo al menos diez días entre la fecha de asignación y la fecha de entrega. Las evaluaciones de los trabajos también retroalimentaban a los estudiantes en relación a cómo mejorar su trabajo escrito de acuerdo a los criterios pautados en las rúbricas.

En el primer trabajo, el estudiante debía desarrollar un ensayo sobre Ingeniería Telemática. Los estudiantes tenían que hacer una investigación sobre las diferentes áreas que componen el campo y sintetizar 
la información en cinco párrafos. Debían mostrar una conceptualización del tema y conectar las diferentes ideas de forma coherente. El segundo trabajo, tenía el propósito de expandir la conceptualización e incorporaba mayores temas a ser conectados. La temática en este caso es del Internet de las Cosas y los estudiantes debían elaborar un ensayo que conectara las diferentes tecnologías que componen el tema y su relación con el desarrollo de la sociedad.

El tercer y cuarto trabajo desarrollaron un artículo de forma grupal y con criterios a evaluar de acuerdo a la lista de cotejo entregada:

Tabla 1: Criterios de evaluación de trabajo escrito

\begin{tabular}{|l|l|l|l|}
\hline \multicolumn{1}{|c|}{ Criterios } & Logrado & $\begin{array}{c}\text { Medianamente } \\
\text { logrado }\end{array}$ & No logrado \\
\hline $\begin{array}{l}\text { Introduce el tema y explica los aspectos } \\
\text { más importantes. }\end{array}$ & & & \\
\hline $\begin{array}{l}\text { Contenido: Detalla todo el contenido } \\
\text { sin ser redundante. Organiza de forma } \\
\text { lógica los contenidos. Une las diferentes } \\
\text { informaciones de forma fluida. }\end{array}$ & & & \\
\hline $\begin{array}{l}\text { Analiza con profundidad y elabora } \\
\text { conclusiones del tema. }\end{array}$ & & & \\
\hline $\begin{array}{l}\text { La escritura es coherente y con pocos } \\
\text { errores gramaticales. }\end{array}$ & & & \\
\hline $\begin{array}{l}\text { El texto posee un uso apropiado de } \\
\text { referencias }\end{array}$ & & & \\
\hline
\end{tabular}

El tercer trabajo desarrolló el tema de los protocolos de comunicación utilizados en el Internet de las Cosas, donde los estudiantes deben demostrar un entendimiento de los diferentes protocolos, como se relacionan entre sí y cuándo es más apropiado utilizar unos en lugar de otros. Para el último trabajo, los estudiantes abordaron el tema de plataformas de hardware y sistemas operativos utilizados en el Internet de las Cosas. Se espera que los estudiantes puedan comparar las distintas plataformas de hardware y los distintos sistemas operativos. Al mismo tiempo, tienen que poder identificar las combinaciones más apropiadas entre las plataformas de hardware y los sistemas operativos, indicando bajo qué aplicación es más apropiada una combinación en específico.

En cada asignación se estableció de forma explícita los criterios a evaluar para asegurar que los estudiantes supieran qué se esperaba en el reporte y cómo sería calificado. Se utilizó el reporte final del proyecto para evaluar el desarrollo de la comunicación escrita de los estudiantes. La comunicación oral se desarrolló con la exposición oral de los trabajos realizados durante el periodo académico. Cada estudiante tuvo la oportunidad de presentar sus dos trabajos escritos de forma individual, y de forma grupal presentaron el primer trabajo. En ambas presentaciones los estudiantes recibieron una retroalimentación de las mismas a partir de una rúbrica de exposición oral, que fue utilizada para evaluar la presentación final, la cual se utilizó como referencia para evaluar el desarrollo de la comunicación oral de los estudiantes.

En paralelo con los módulos de la asignatura, los estudiantes desarrollaron un proyecto en grupo a lo largo del periodo académico. Los estudiantes tenían que identificar una necesidad y desarrollar un dispositivo que se conectara al internet y brindara una solución a dicha problemática. El proyecto tenía como requisito:

- Utilizar la plataforma Arduino para desarrollar un dispositivo que se conectara al internet

- Integrar los componentes electrónicos que se necesitasen

- Integrar los componentes de software que se necesitasen

Para formar los equipos utilizamos las sugerencias revisadas para formación de equipos de Mathieu, 
Tannenbaum, Donsbach, y Alliger (2014). Creamos grupos heterogéneos dentro de nuestras posibilidades dado que todos los estudiantes pertenecen a la carrera de Ingeniería Telemática. Cada grupo tuvo estudiantes que estaban a diferentes niveles en ingeniería, por ejemplo, al menos un integrante que había cursado una asignatura de electricidad y/o una asignatura de ciencias de computación; igualmente, al menos un estudiante que no había cursado ninguna asignatura relacionada. Por la desproporción en el género de los estudiantes, cada grupo tenía una representación femenina. Se formaron cuatro grupos de cuatro estudiantes y uno de cinco.

Una vez los grupos estuvieron establecidos, decidieron un nombre que los identificaría. Catorce días después debían identificar un problema y definir el proyecto que desarrollarían. Cuando los proyectos estuvieron definidos, cada grupo debió establecer los roles y responsabilidades de cada integrante. En el foro y en las horas de clase iban exponiendo los avances de los proyectos y los mecanismos utilizados como grupo para llegar al estado de avance. El profesor tenía la responsabilidad de guiar a los estudiantes en el proceso de desarrollo del proyecto y promover el desarrollo de las competencias orales y escritas para presentar dicho proyecto.

Al finalizar el curso, se suministró una encuesta en línea de 10 preguntas utilizando la escala Likert de 5 puntos. El propósito de la encuesta era evaluar la satisfacción del estudiante con la metodología de proyecto utilizada.

Preguntas de la encuesta:

1. ¿La metodología de enseñanza-aprendizaje utilizada en esta asignatura es adecuada a la forma en que me gusta estudiar?

2. ¿La metodología de enseñanza-aprendizaje utilizada permite que demuestre mis habilidades?

3. ¿Logré más en esta asignatura de lo que inicialmente pensé que podía?

4. ¿Disfruté trabajar en proyectos auténticos?

5. ¿Logré satisfacer los requerimientos de un proyecto tan demandante?

6. ¿Los recursos expuestos en el curso me permitieron satisfacer los requerimientos del proyecto?

7. ¿Tengounbuenentendimientodecómoutilizarla plataformaArduino para crear proyectos de loT?
8. ¿Prefiero que el profesor asigne los temas del proyecto?

9. ¿Los trabajos puestos y la retroalimentación me ayudaron a concentrarme en lo que debo lograr?

10. ¿Me hubiera gustado que la asignatura no contemplara un proyecto como forma de evaluación?

\section{Resultados y discusión}

Todos los proyectos realizados cumplieron con los requisitos mínimos y su complejidad fue similar, aunque utilizaron diferentes vías para lograr la conectividad hacia internet. La problemática a resolver por cada grupo fue diferente.

El primer grupo se encargó de eficientizar el control de acceso de iluminación de un hogar; el segundo grupo desarrolló una aplicación para poder ver el estado actual de dos parqueos utilizando sensores del tipo ultrasonido. El tercer grupo desarrolló un sistema de alarma que notifica por una aplicación al usuario cuándo una persona no deseada entra al espacio asignado para monitorear la alarma, este sistema tiene una capacidad de hasta cuatro usuarios.

El cuarto grupo desarrolló un sistema automatizado para mantener el nivel apropiado de humedad de la tierra en un jardín, comunicando al usuario por una aplicación el estado actual del jardín en tiempo real. El quinto grupo presentó un sistema que permite administrar de forma remota el encendido y apagado de cualquier equipo electrónico conectado a su solución utilizando la red móvil.

\section{Comunicación escrita}

En la habilidad de comunicación escrita se pudo observar en el transcurso del periodo académico una mejora de los trabajos asignados. La tabla 2 muestra el promedio y la desviación estándar de los trabajos corregidos.

La disminución de la desviación estándar evidencia una homogenización de las capacidades de comunicación escrita de los estudiantes en la asignatura. Además, el incremento del promedio muestra que están más acoplados a la rúbrica de evaluación.

Con relación al reporte escrito del proyecto final, el promedio fue de 76.29 con una desviación estándar de 4.23. Los grupos presentaron debilidades comunes en la contextualización de la problemática; la introducción 
Tabla 2: Promedio y desviación estándar de trabajos entregados

\begin{tabular}{|c|c|c|c|c|}
\hline & Trabajo 1 & Trabajo 2 & Trabajo 3 & Trabajo 4 \\
\cline { 2 - 5 } & 83 & 70 & 76 & 83 \\
\hline $\begin{array}{c}\text { Promedio } \\
\text { estándar }\end{array}$ & 20 & 30 & 9 & 6 \\
\cline { 2 - 5 } & & & 9 & 6 \\
\hline
\end{tabular}

al tema fue poco atractiva y rara vez se realizó un desglose del contenido del proyecto. Otra debilidad común se encontró en el uso del formato apropiado dentro del texto y en la bibliografía. En adición, dentro del texto los argumentos utilizados por los estudiantes pudieron ser mejores si hubieran utilizado referencias de mayor relevancia.

Tabla 3: Logros medidos en el trabajo escrito final por criterio de evaluación

\begin{tabular}{|c|c|}
\hline Criterios & Nivel de logro \\
\hline Introduce el tema y explica los aspectos más importantes. & Introducción poco atractiva. \\
\hline $\begin{array}{l}\text { Contenido: Detalla todo el contenido sin ser redundante. } \\
\text { Organiza de forma lógica los contenidos. Une las diferentes } \\
\text { informaciones de forma fluida. }\end{array}$ & $\begin{array}{l}\text { Rara vez presentó un desglose } \\
\text { de lo que se iba a tratar. }\end{array}$ \\
\hline Analiza con profundidad y elabora conclusiones del tema. & $\begin{array}{l}\text { Los argumentos pudieron ser } \\
\text { mejores. }\end{array}$ \\
\hline La escritura es coherente y con pocos errores gramaticales. & Presenta aún cierta debilidad. \\
\hline El texto posee un uso apropiado de referencias & $\begin{array}{l}\text { Debieron utilizar referencias de } \\
\text { mayor relevancia. }\end{array}$ \\
\hline
\end{tabular}

Se pudo notar un buen desempeño en el desarrollo de la metodología, los grupos pudieron detallar sin ser redundantes los pasos para elaborar su proyecto. Sin embargo, las justificaciones fueron débiles por la falta de referencia relevante. Los resultados del proyecto fueron plasmados de forma apropiada y la mayoría de los grupos pudo hacer comparaciones del desempeño en el proyecto.

\section{Comunicación oral}

Fue en la presentación de los proyectos donde se logró la mayor evolución de los estudiantes. Se pudo demostrar un buen dominio del tema en la exposición de los trabajos. La comunicación de los estudiantes fue fluida en su mayoría cuando expusieron sus temas y en la sección de preguntas y respuestas. Sin embargo, en el manejo del tiempo hubo una diferencia entre los distintos grupos. Tres de los cinco grupos pudieron exponer la totalidad del proyecto dentro del tiempo asignado, dos grupos utilizaron un 30\% adicional del tiempo asignado. Hacer exposiciones durante el periodo académico y ofrecerles retroalimentaciones en público permite que todos los 
estudiantes desarrollen pautas sobre las mejores prácticas para la comunicación oral. En la evaluación de las presentaciones, el promedio obtenido fue de 91.71 con una desviación estándar de 5.84

\section{Trabajo en equipo}

De los 21 estudiantes inscritos, solo un estudiante no colaboró con el equipo al mismo nivel que el resto de los integrantes. La evaluación de pares arrojó un promedio de 87.00 con una desviación estándar de 17.81 y la autoevaluación arrojó un promedio de 88.09 con una desviación estándar de 17.76, (tabla 4). Se puede determinar que cada integrante fue objetivo al nivel de colaboración que aportó dentro del grupo. El uso de una rúbrica de evaluación de trabajo en equipo a ser llenada por los integrantes como una evaluación por pares puede permitir identificar el nivel de colaboración de cada integrante. Esto facilita la evaluación final del proyecto por parte del profesor.

Tabla 4: Resultado de la evaluación por pares y la autoevaluación

\begin{tabular}{|c|c|c|}
\hline & Evaluación por pares & Autoevaluación \\
\hline Promedio & 87.00 & 88.09 \\
\hline Desviación estándar & 17.81 & 17.76 \\
\hline
\end{tabular}

Dentro de la encuesta suministrada al finalizar el curso, se tomaron preguntas sobre el trabajo en equipo. El $85.7 \%$ de los estudiantes mostró preferencia en realizar un trabajo en grupo como forma de evaluación de la asignatura. Un $90.5 \%$ estaba conforme con el tamaño de los grupos.

\section{Satisfacción de los estudiantes con la metodología utilizada}

El $100 \%$ de los estudiantes mostró un nivel de satisfacción positiva a la metodología de enseñanza aprendizaje utilizada. Los estudiantes indicaron que pudieron lograr más en la asignatura que lo inicialmente esperado. Todos los estudiantes acordaron que el uso de proyectos relevantes a problemáticas reales permitió que se interesaran más en la asignatura y en la carrera. El nivel de dificultad de los proyectos y los recursos expuestos en el curso permitieron que los estudiantes pudieran desarrollar los distintos proyectos y solucionar las problemáticas encontradas.

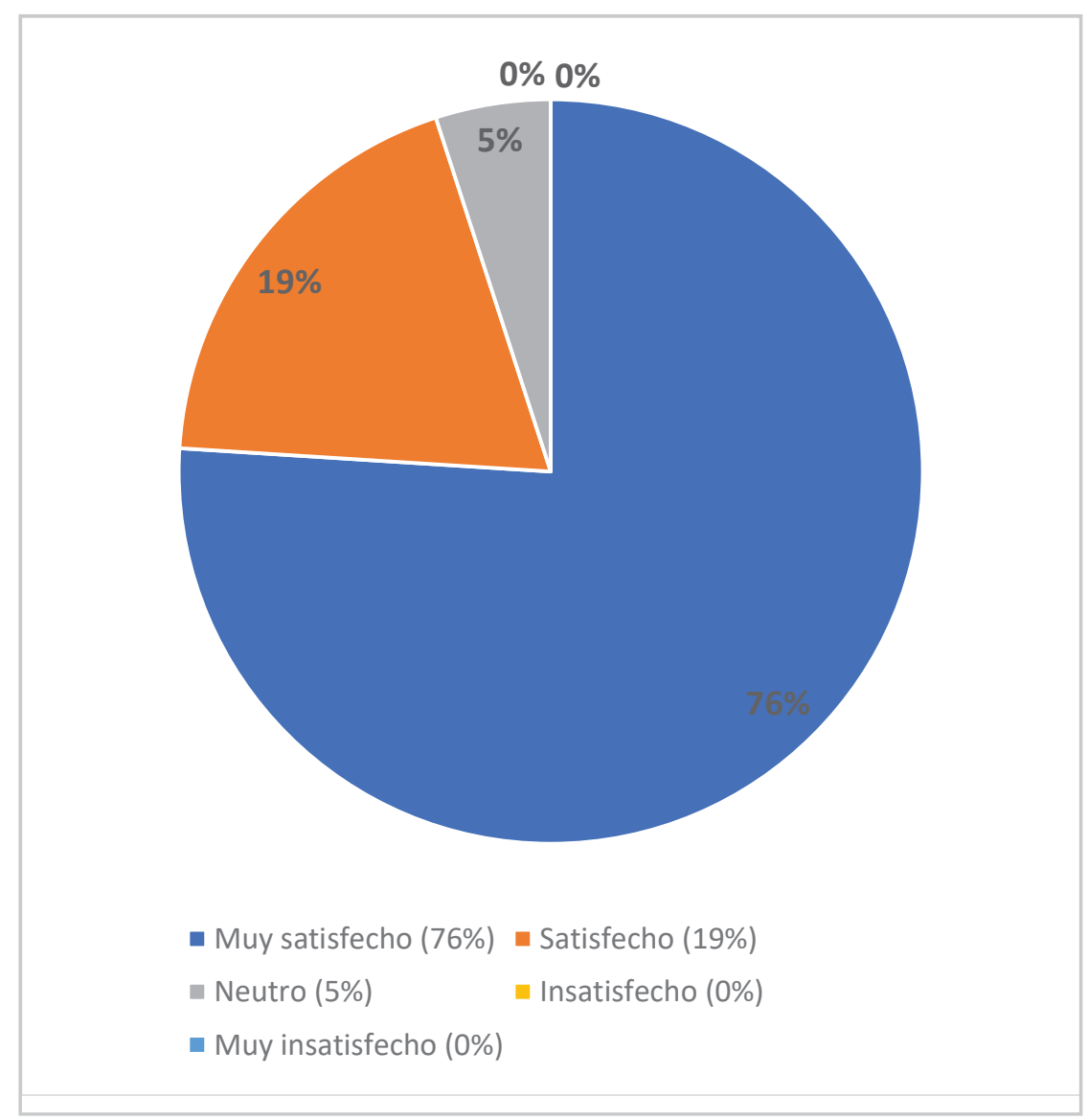

Figura: Nivel de satisfacción del estudiante en el logro de su proyecto 


\section{Conclusión}

La práctica de la ingeniería requiere una combinación de competencias técnicas y habilidades blandas. Un ingeniero debe poder comunicarse con diferentes actores para exponer su idea o proyecto. Al mismo tiempo, este debe poder desarrollar e integrar los conceptos de ingeniería para solucionar un problema.

Se utilizó la metodología de Aprendizaje Orientado a Proyectos para crear un proyecto relacionado al Internet de las Cosas y conectar las capacidades técnicas con habilidades blandas como trabajo en equipo y comunicación oral y escrita. Para medir el desarrollo de las habilidades blandas se utilizaron rúbricas que fueron presentadas y socializadas con los estudiantes desde el primer día de la asignatura.

El uso de la metodología mencionada en conjunto con un Sistema de Gestión del Aprendizaje permitió el desarrollo de las habilidades blandas de los estudiantes. Se puede notar una mejora significativa en su escritura para reportes técnicos y en la habilidad de presentar y defender su proyecto. Estas habilidades se deben seguir desarrollando durante su trayectoria en la Universidad.

Sería interesante seguir midiendo el rendimiento de estos estudiantes en diferentes trayectorias de su vida universitaria y medir cómo el uso de estas estrategias que hemos implementado aplicadas en otras asignaturas contribuirían a una mejora en las presentaciones de los Trabajos de Fin de Grado. También, se pudiera comparar las competencias de las habilidades blandas de los estudiantes que han sido expuestos a estas estrategias con los estudiantes de años anteriores o con grupos controles para verificar el nivel de dominio en el desarrollo de dichas habilidades.

\section{Referencias}

Alvarez, M. \& Boillos, M. (2015). La competencia comunicación escrita. En Lourdes VillardónGallego, Competencias genéricas en educación superior. Metodologías específicas para su desarrollo. España: Narcea.

Calnon, M., Gifford, C. M., \& Agah, A. (2012). Robotics competitions in the classroom: Enriching graduate-level education in computer science and engineering. Global Journal of Engineering Education, 14(1), 6-13.

Dahm, K. D., Farrell, S., \& Ramachandran, R. P. (2015). Communication in the Engineering Curriculum: Learning to Write and Writing to Learn. Journal of Engineering Education Transformations, 29(2), 1-8.

Davcev, D., Stojkoska, B., Kalajdziski, S., \& Trivodaliev, K. (2016, June 23). Project Based Learning of Embedded Systems. arXiv [cs.OH]. Retrieved from http://arxiv.org/abs/1606.07498

David, H. (2015). Why are there still so many jobs? The history and future of workplace automation. The Journal of Economic Perspectives: A Journal of the American Economic Association, 29(3), 3-30.

Deming, D. J. (2015). The growing importance of social skills in the labor market. The Quarterly Journal of Economics. Retrieved from https://academic. oup.com/qje/article-abstract/doi/10.1093/qje/ qjx022/3861633/The-Growing-Importance-ofSocial-Skills-in-the

DePiero, F. W., \& Slivovsky, L.A. (2007). Multidisciplinary experiencesforundergraduateengineeringstudents. University "Politehnica" of Bucharest Scientific Bulletin, Series C: Electrical Engineering, 10.

Felder, R. M. (1993). Reaching the second tier. Journal of College Science Teaching, 23(5), 286-290.

Frey, C. B., \& Osborne, M. A. (2017). The future of employment: How susceptible are jobs to computerisation? Technological Forecasting and Social Change, 114, 254-280.

Hernando, M., Galan, R., Navarro, I., \& RodriguezLosada, D. (2011). Ten Years of Cybertech: The Educational Benefits of Bullfighting Robotics. IEEE Transactions on Education, 54(4), 569-575.

Jadhav, P. S., Mulla, A. M., \& Jadhav, P. M. (2017). Blending ICT with Project Based Learning for Effective Teaching and Learning of Mechatronics. Journal of Engineering Education Transformations, 30(3), 47-51. 
Krithivasan, S., Shandilya, S., Arya, K., Lala, K., Manavar, P., Patii, S., \& Jain, S. (2014). Learning by competing and competing by learning: Experience from the e-Yantra Robotics Competition. In 2014 IEEE Frontiers in Education Conference (FIE) Proceedings (pp. 1-8). ieeexplore.ieee.org.

Lou, Y., \& Kim MacGregor, S. (2004). Enhancing Project-Based Learning Through Online BetweenGroup Collaboration. Educational Research and Evaluation: An International Journal on Theory and Practice, 10(4-6), 419-440.

Maffioli, F. \& Giuliano, A. (2003). Tuning Engineering education into the European higher education orchestra. European Journal of the Engineering Education. (251-273).

Mathieu, J. E., Tannenbaum, S. I., Donsbach, J. S., \& Alliger, G. M. (2014). A review and integration of team composition models: Moving toward a dynamic and temporal framework. Journal of Management, 40(1), 130-160.

Mills, J. E. \& Treagust, D. F. (2003). Engineering education-ls problem-based or project-based learning the answer. Australasian Journal of Engineering Education, 3(2), 2-16.

Murti, A. B. (2014). Why soft skills matter. IUP Journal of Soft Skills, 8(3), 32-36. Retrieved from https:// search.proquest.com/docview/1627986081?accou ntid $=139608$

Nafea, S. M., Maglaras, L. A., Siewe, F., \& Shehab, M. E. (2015). A novel adaptive learning management system using ontology. In 2015 IEEE Seventh International Conference on Intelligent Computing and Information Systems (ICICIS) (pp. 328-335). ieeexplore.ieee.org.

Neebel, D. J., Merkel, C., \& Wong, A. (2013). Engaging a variety of students in digital design with competition. In 2013 IEEE Frontiers in Education Conference (FIE) (pp. 1091-1095). ieeexplore. ieee.org.

Ortega, C.E., Febles, J.P., \& Estrada, V. (2016). Una estrategia para la formación de competencias blandas desde edades tempranas. Revista Cubana de Educación Superior, 35(2), 35-41. Recuperado en 04 de diciembre de 2017, de http://scielo.sld.cu/scielo.php?script=sci_ arttext\&pid=S0257-43142016000200003\&Ing= es\&tIng=es.

Palma, M., Miñán, E. \& Rios, I. (2011). Competencias Genéricas en Ingeniería: un Estudio Comparado en el Contexto Internacional. XV Congreso Internacional de Ingeniería de Proyectos, 6-8 julio de 2011. Huesca, España. pp. 2552-2569

Pavlakis, A., \& Kelley, C. (2016). Accreditation in the professions: Implications for educational leadership preparation programs. Journal of Research on Leadership Education, 11(1), 68-90.

Porter, L., \& Simon, B. (2013). Retaining Nearly Onethird More Majors with a Trio of Instructional Best Practices in CS1. In Proceeding of the 44th ACM Technical Symposium on Computer Science Education (pp. 165-170). New York, NY, USA: ACM.

Robles, M. M. (2012). Executive perceptions of the top 10 soft skills needed in today's workplace. Business Communication Quarterly, 75(4), 453-465.

Sharma, B., Steward, B., Ong, S. K., \& Miguez, F. E. (2016). Development of resource modules to enhance project based learning in an interdisciplinary engineering course. In 2016 ASABE Annual International Meeting (p. 1). American Society of Agricultural and Biological Engineers.

Yalvac, B., Smith, H. D., Troy, J. B., \& Hirsch, P. (2007). Promoting advanced writing skills in an upperlevel engineering class. Journal of Engineering Education, 96(2), 117.

Yam, S., \& Rossini, P. (2010). Implementing a projectbased learning approach in an introductory property course. PRRES. Retrieved from http://www. prres.net/Proceedings/..\%5CPapers $\% 5$ CYam_ Implementing_a_Project-Based_Learning_ Approach_in_an_Introductory_Property_Course. pdf 